\title{
Improving availability and accuracy of GPS/BDS positioning using QZSS for single receiver
}

\author{
Zengke $\mathrm{Li}^{1} \cdot \mathrm{Fu} \mathrm{Chen}^{2}$
}

Received: 23 September 2015/ Accepted: 2 March 2016/Published online: 14 March 2016

(C) Akadémiai Kiadó 2016

\begin{abstract}
The Quasi-Zenith Satellite System (QZSS) service area covers East Asia and Oceania region and its platform is multi-constellation GNSS. The QZSS system is not required to work in a stand-alone mode, but together with data from other GNSS satellites. QZSS data is processed and analysed for single receiver together with GPS/BDS data in the paper. Single point positioning mode, static precise point positioning mode and kinematic precise point positioning mode are used to assess the impact of QZSS on GPS/ BDS single receiver positioning. The data corresponding to the day 2015-02-05 taken from the IGS station of CUT0 is considered. The sky plots and number of satellite for the various satellite systems are given. The PDOP (Position Dilution of Precision) value, position error and solution success rate under different cut-off elevation angles are compared between GPS/BDS and GPS/BDS/QZSS. The results indicate that QZSS is able to decrease the position error and increase success rate of resolution for GPS/BDS single receiver positioning, especially under high cut-off elevation angle. The availability and accuracy of GPS/BDS positioning are improved using QZSS for single receiver.
\end{abstract}

Keywords Quasi-Zenith Satellite System · Single point positioning · Precise point positioning

\section{Introduction}

The Quasi-Zenith Satellite System (QZSS) is the Japanese regional satellite navigation system which provides positioning and timing information to users ( $\mathrm{Li}$ and Rizos 2011). The Quasi-Zenith Satellite System service area covers East Asia and Oceania region.

Fu Chen

chenfu_cumt@yeah.net

1 School of Environment and Spatial Informatics, China University of Mining and Technology, Xuzhou, China

2 Low Carbon Energy Institute, China University of Mining and Technology, Xuzhou, China 
Recently, QZSS has been developed to enhance the performance of GNSS positioning, navigation and timing services.

Some research about QZSS has been carried out in recent years. The achievable performance of the GPS augmentation using QZSS were obtained using software simulation in 2004. The simulation analysis indicated that QZSS does not only effectively increase the availability and accuracy of GPS positioning, but also improve the reliability of GPS positioning in Japan and its neighbouring area (Wu et al. 2004). GPS availability and DOP performance were analysed in Seoul city center combining a geostationary satellite as well as QZSS with the conventional GPS. Using QZSS, a higher level of availability and more accurate user position were provided than when using GPS with a geostationary satellite (Yoo et al. 2009). Additions of QZSS to GPS, the average of the GPS/QZSS positioning solution horizontal error was $0.58 \mathrm{~m}$, which shown an improvement of $52.8 \%$ than the GPS-only solution (Li and Rizos 2011). The inclusion of the QZSS satellite in the rover has shown reductions of the order of $20 \%$ in convergence time in the Asia Pacific region. At the same time the inclusion of the QZSS satellite represented an accuracy improvements of approximately $10 \%$ in horizontal and $25 \%$ in vertical (Landau et al. 2012). According to real-time positioning simulation, the navigation by QZSS could obtain horizontal accuracy of $10 \mathrm{~m}$ or better and vertical accuracy of $14 \mathrm{~m}$ or better with moderate user range error (Yamada et al. 2012). The carrier-to-noise density ratios from QZSS and GPS have been compared for a receiver in Chofu, Japan. Compared to GPS, the path loss of QZSS varied by about $1.5 \mathrm{~dB}$ because of the eccentricity of the orbit (Hauschild et al. 2012). With the GLONASS and QZSS augmentation to GPS-only processing shown improvements both for kinematic coordinate and ZTD solutions, especially in the period with lower number of GPS satellites (Iwabuchi et al. 2013). The RTK solutions during $24 \mathrm{~h}$ by GPS and GPS + QZSS observations shown that the resolutions were slightly better with one additional QZSS satellite augmentation (Lukes et al. 2013). The correction messages, such as orbits and clock information, could be delivered by the LEX signal of QZSS, which was use to realize precise point positioning (PPP) for real-time applications. The achievable PPP positioning accuracy in real-time using the current LEX corrections message could achieve the accuracy of decimetre level (Choy et al. 2013). Observations from the Chinese BeiDou Navigation Satellite System (BDS), European Galileo, American Global Positioning System (GPS) and the Japanese Quasi-Zenith Satellite System (QZSS) were combined to realize long baseline RTK positioning (Odolinski et al. 2014), 3D building model-based pedestrian positioning method (Hsu et al. 2015) and single-frequency RTK (Odolinski et al. 2015) and to analyse the robustness of single-frequency instantaneous carrier-phase attitude determination (Nadarajah and Teunieesn 2014) and receiver autonomous integrity monitoring (RAIM) performances (Su et al. 2014). L5-observables of multi-constellation GNSS formed from IRNSS, GPS, Galileo, and QZSS were evaluated for real-time kinematic positioning using the standard LAMBDA method. For singlefrequency carrier phase-based positioning and navigation, the results shown better ambiguity resolution performance of L5/E5a-only processing than that of L1/E1-only processing (Nadarajah et al. 2015). Regional orbits and clocks for real-time PPP would be generated and delivered through the QZSS LEX signal. The real time PPP solutions using the developed 'Australian LEX' system have comparable performance to those obtained from the IGS real-time products through a land network, showing that latency and reliability of the system is adequate for real-time PPP (Harima et al. 2014). A new positioning method improving the availability and accuracy of Multi-global navigation satellites system (GNSS) positioning by using QZSS satellites in urban canyon environments was 
proposed. QZSS was regarded as the sole master satellite and the ambiguity of QZSS is fixed by the wide-lane method and the QZSS LEX signal (Kitamura et al. 2014).

In the present study, QZSS is used to improve the availability and accuracy of GPS/ BDS single receiver positioning. The observation is process by single point positioning (SPP), static precise point positioning (PPP) and kinematic precise point positioning mode. The paper is divided into 5 sections. Following this introduction, the introduction of QZSS is overviewed in Sect. 2. Section 3 describes GPS/BDS positioning with QZSS, including SPP, static PPP and kinematic PPP. Test results are then presented and analysed in Sect. 4, followed by a summary of the main conclusions.

\section{Quasi-Zenith Satellite System}

The Quasi-Zenith Satellite System (QZSS), is a developing regional time transfer system and satellite based augmentation system for the Global Positioning System, which would be receivable within East Asia and Oceania region. The first satellite 'Michibiki' was launched on 11 September, 2010. After confirmation of the stability to provide specified performance in IS-QZSS, L1 C/A and L2C signals were set healthy on 22 June, 2011 and L5 and L1C signals were set healthy on 14 July, 2011. Four satellites constellation will be constructed and the service will be provided in 2018.

The QZSS aims at improving positioning accuracy of one meter to the centimeter level compared to the conventional GPS error of tens of meters by transmitting support signals. The Quasi-Zenith Satellites transmit signals compatible with the GPS L1C/A signal, as well as the modernized GPS L1C, L2C signal and L5 signals, which can provide seamless PNT (Positioning, Navigation and Timing) services by combining usage with GPS and increase coverage and availability of PNT services even in downtown and mountainous areas. QZSS also transmits two augmentation signals, i.e., L1-SAIF on $1575.42 \mathrm{MHz}$ and LEX on 1278.75 MHz. The LEX signal can enhance GPS performance by transmitting error correction and integrity information.

The QZSS coordinate system is known as the Japan satellite navigation Geodetic System (JGS). This coordinate System is operated so as to approach International Terrestrial (ITRS). The time scale offset with the GPS is less than $2.0 \mathrm{~m}(95 \%)$. The coordinate system offset with GPS is less than $0.02 \mathrm{~m}$. Integer second offset for TAI is the same as GPS, and TAI is $19 \mathrm{~s}$ ahead of QZSST. The SV clocks of Quasi-Zenith Satellite (QZS) and GPS satellites are both controlled with respect to the offset with the GPS time scale (GPST). The Signal-in-Space (SIS) User Range Error is less than $1.6 \mathrm{~m}$ (95\%) without time and coordination offset error. The QZSS orbit parameter and tracking range is indicated in Table 1 and Fig. 1 shows the ground track of the QZSS.

Table 1 QZSS orbit parameter and tracking range

\begin{tabular}{lll}
\hline Orbit parameter & Nominal allocation & Tracking range \\
\hline Semimajor axis & $42,164 \mathrm{~km}$ & 100 \\
Eccentricity & 0.075 & $0.075 \pm 0.015$ \\
Inclination & $40^{\circ}$ & $36-45^{\circ}$ \\
Argument of perigee & $270^{\circ}$ & $270 \pm 2.5^{\circ}$ \\
Central longitude & $136^{\circ}$ & $130-140^{\circ}$ \\
\hline
\end{tabular}


Fig. 1 The ground track of the QZSS

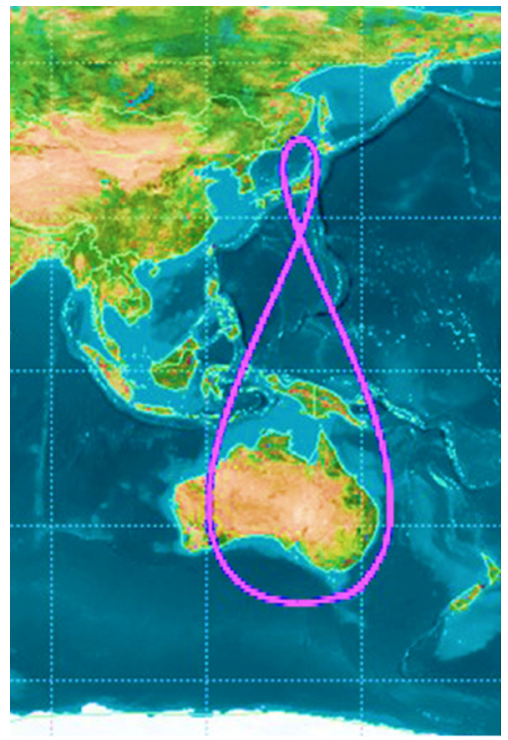

\section{GPS/BDS positioning with QZSS}

The traditional observation model of GNSS positioning, including the pseudorange and carrier phase measurements, can be written as (Du 2010):

$$
\begin{gathered}
P=\rho+c \times\left(d t-d t_{s}\right)+I+T+M_{P}+\varepsilon_{P} \\
\boldsymbol{\Phi}=\rho+c \times\left(d t-d t_{s}\right)-I+T+\lambda N+M_{\Phi}+\varepsilon_{\Phi}
\end{gathered}
$$

where $P$ and $\Phi$ are the pseudorange and carrier phase measurements, respectively. $\rho$ is the geometric distance as a function of receiver and satellite coordinates. $c$ is the speed of light

Fig. 2 Three positioning modes for single receiver

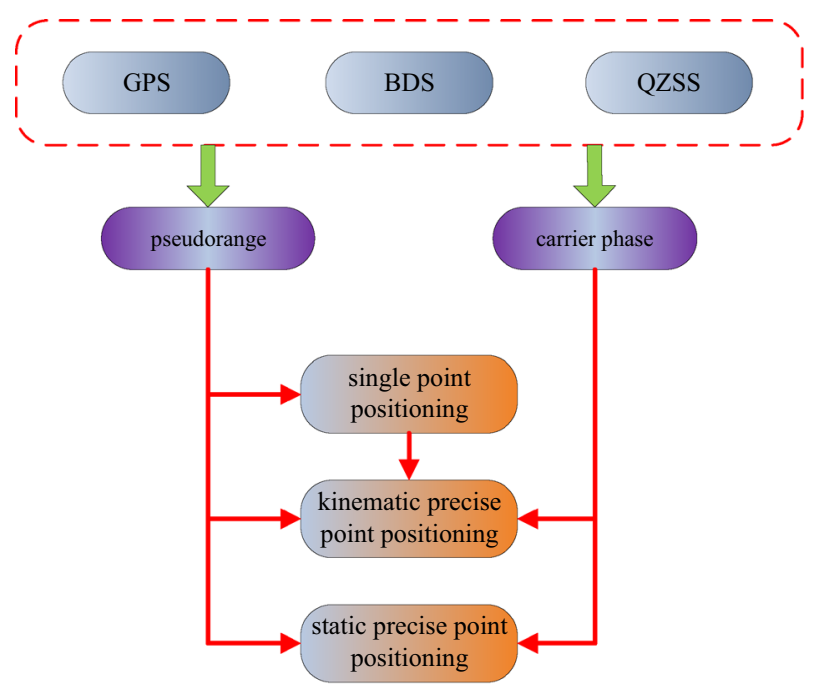




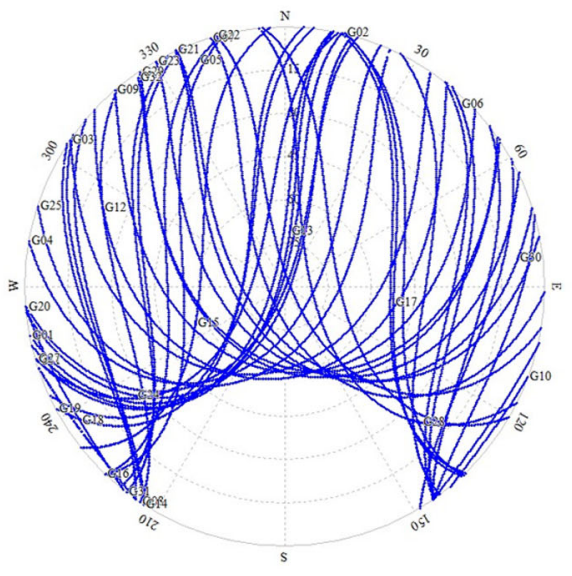

(a)

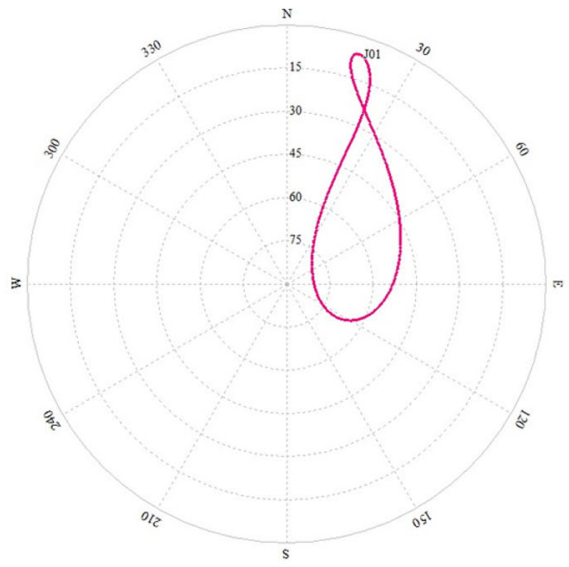

(c)

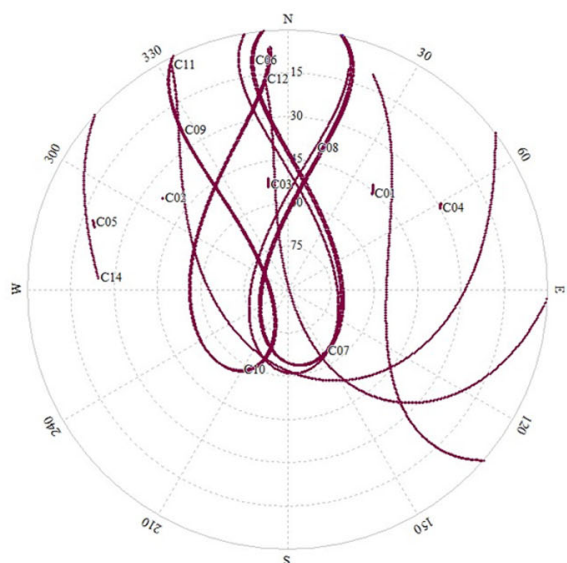

(b)

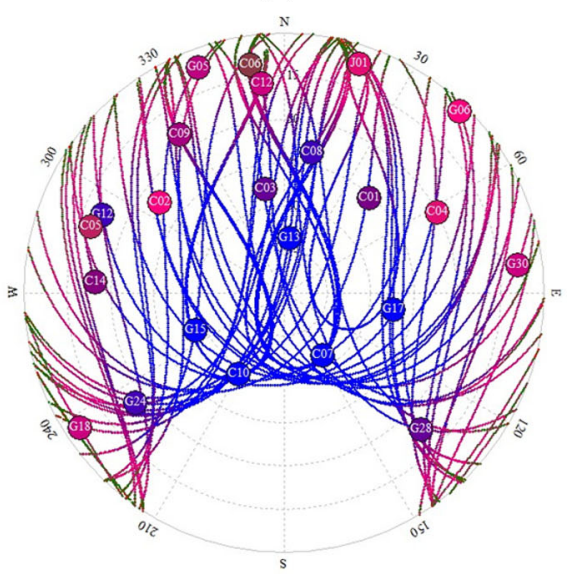

(d)

Fig. 3 Sky plots (azimuth vs. elevation) for the various satellite systems at station CUT0 on February 5, 2015: a GPS; b BDS; c QZSS; d GPS/BDS/QZSS

in vacuum. $d t$ and $d t_{s}$ are the satellite clock error and receiver clock error, respectively. $I$ is the first-order ionospheric delay. $T$ is the tropospheric delay. $M_{P}$ and $M_{\Phi}$ is the multipath error of the pseudorange, carrier phase measurements, $\varepsilon_{P}$ and $\varepsilon_{\Phi}$ are a combination noise of the pseudorange and carrier phase measurements.

In precise point positioning, the uncertainties in the satellite orbit and clock corrections can be significantly reduced by using precise GPS orbit and clock products. The other error sources including the satellite antenna phase centre offset, phase wind up, earth tide, ocean tide loading and atmosphere loading can be eliminated by correction model. The widely used ionosphere-free combination makes use of GPS radio frequency's dispersion property to mitigate the first order ionospheric delay effect. The observation model of ionospherefree combination can be written (Abdel-salam 2005): 


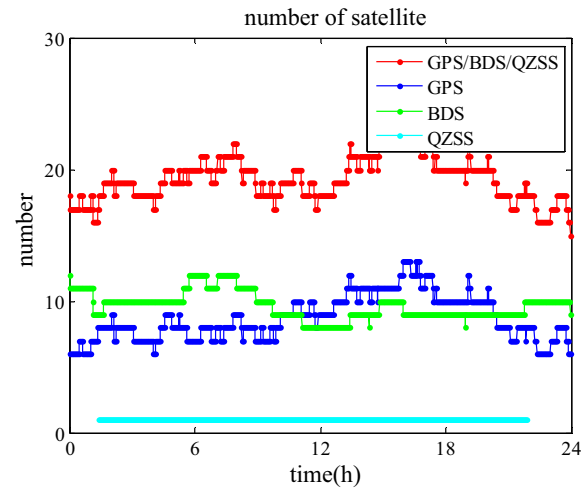

(a)

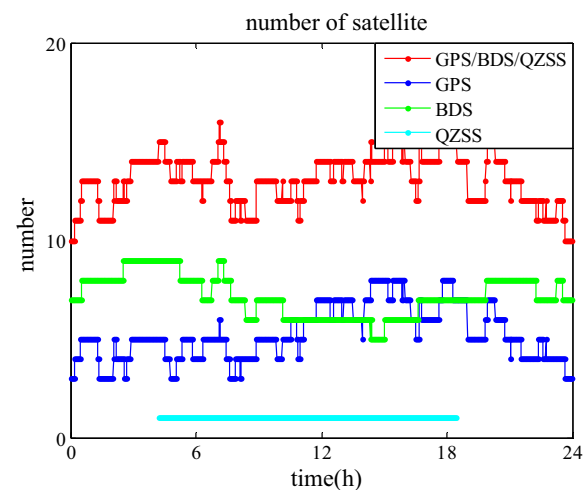

(c)

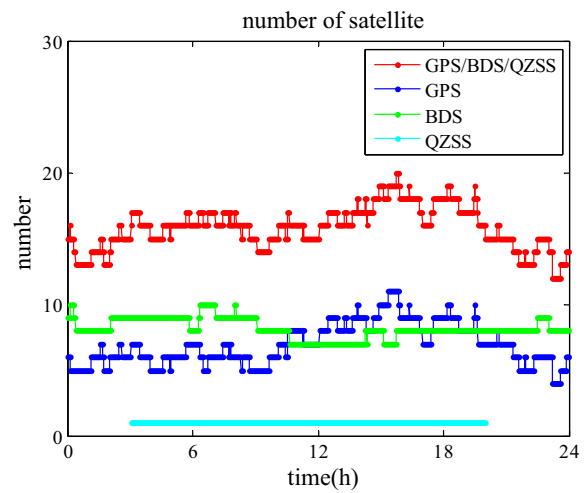

(b)

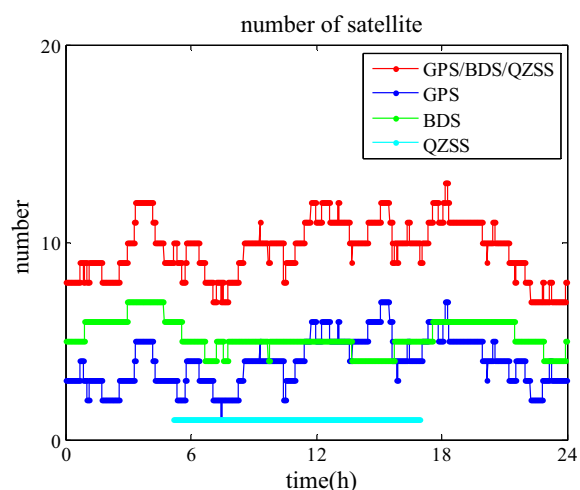

(d)

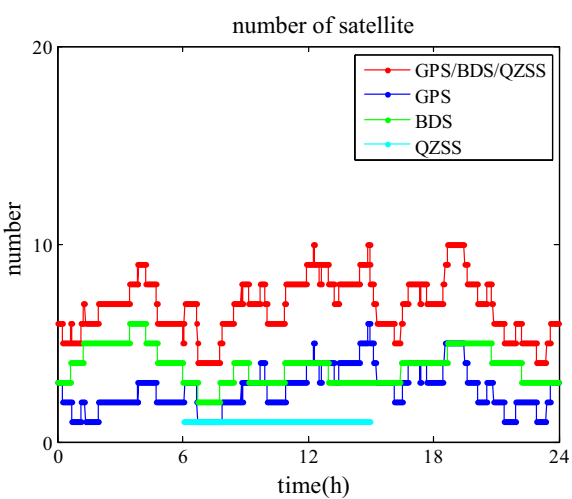

(e)

Fig. 4 The number of satellite for the various satellite systems under different cut-off elevation angles at station CUT0 on February 5, 2015: a $10^{\circ}$; b $20^{\circ}$; c $30^{\circ}$; d $40^{\circ}$; e $50^{\circ}$

$$
P_{i f}=\frac{f_{1}^{2}}{f_{1}^{2}-f_{2}^{2}} P_{1}-\frac{f_{2}^{2}}{f_{1}^{2}-f_{2}^{2}} P_{2}=\rho+c \times\left(d t-d t_{s}\right)+T+M_{P}+\varepsilon_{P}
$$




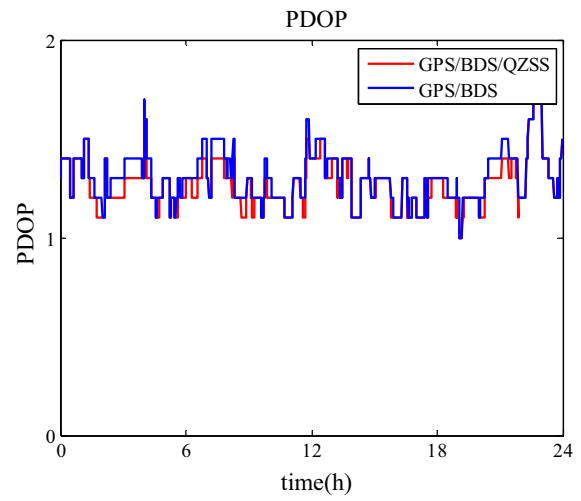

(a)

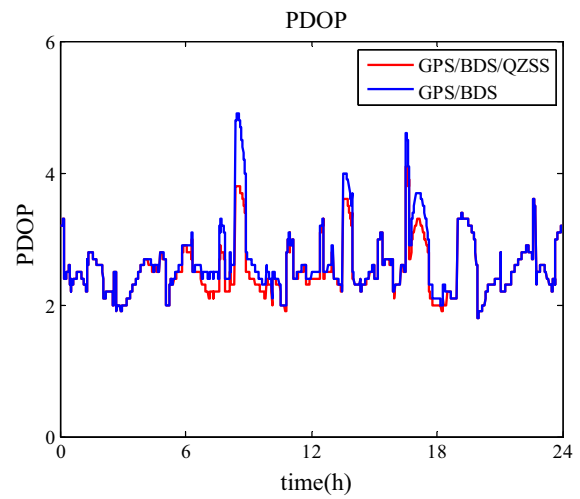

(c)

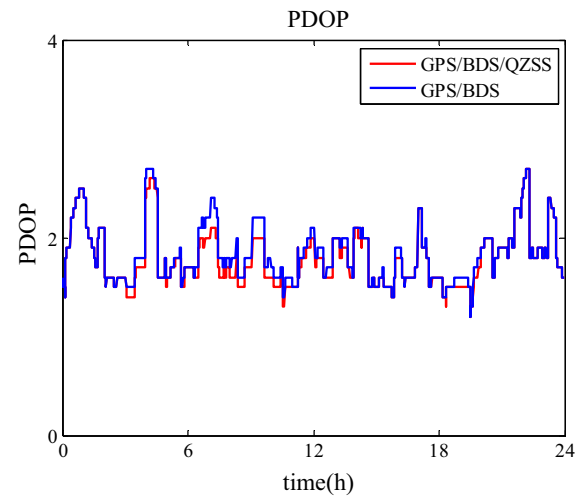

(b)

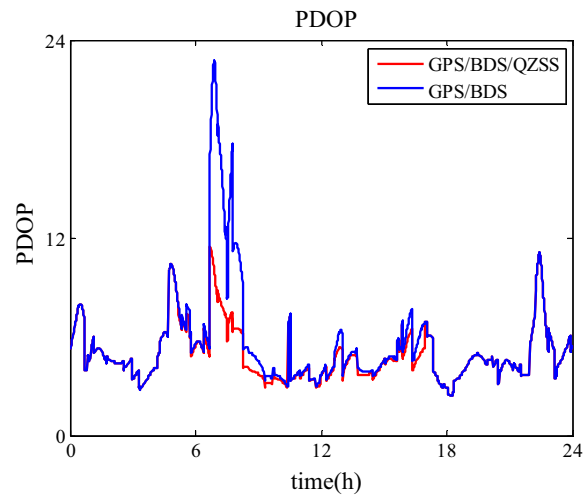

(d)

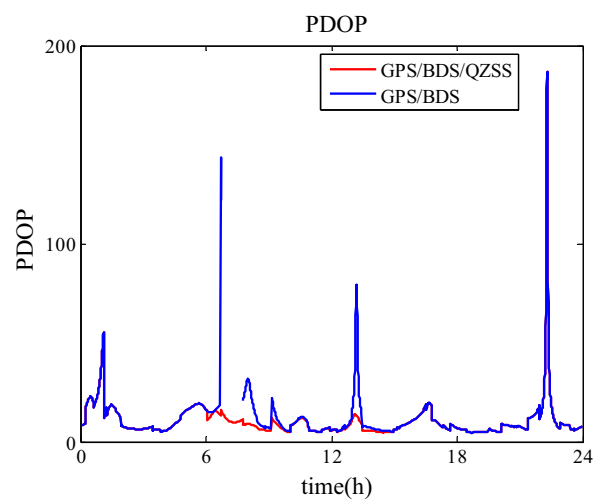

(e)

Fig. 5 PDOP value under different cut-off elevation angles at station CUT0 on February 5, 2015: a 10 b $20^{\circ} ;$ c $30^{\circ} ; \mathbf{d ~} 40^{\circ} ;$ e $50^{\circ}$ 


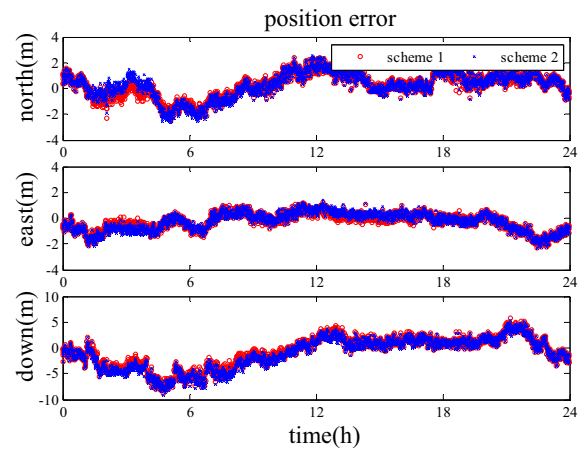

(a)

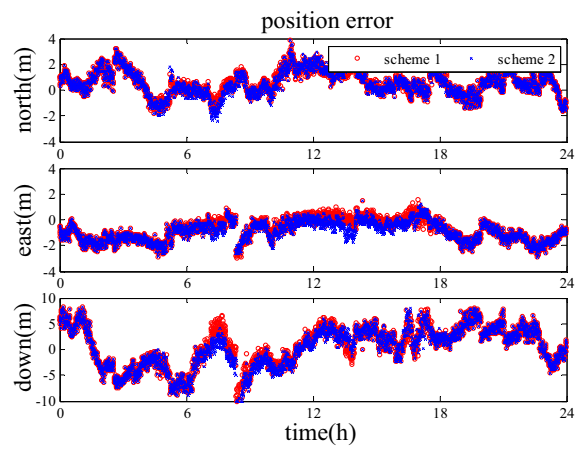

(c)

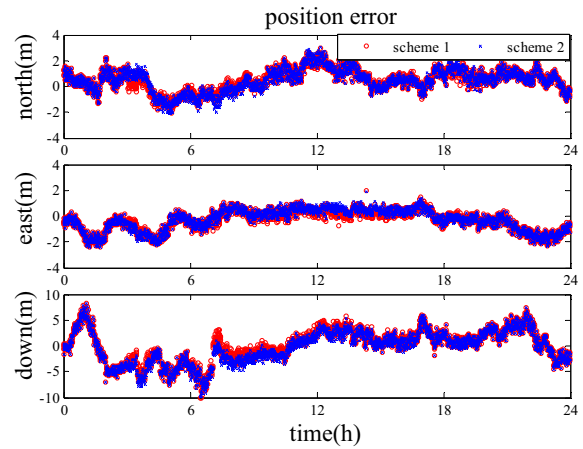

(b)

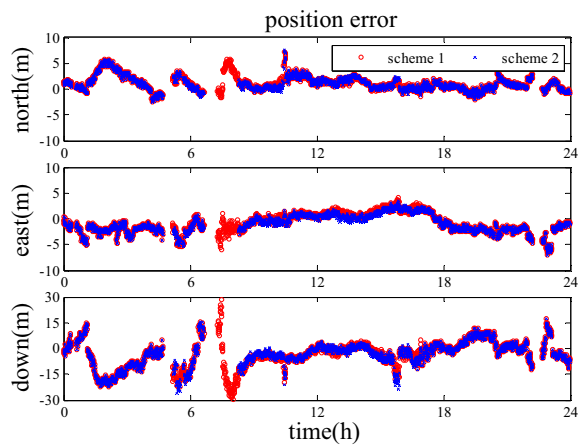

(d)

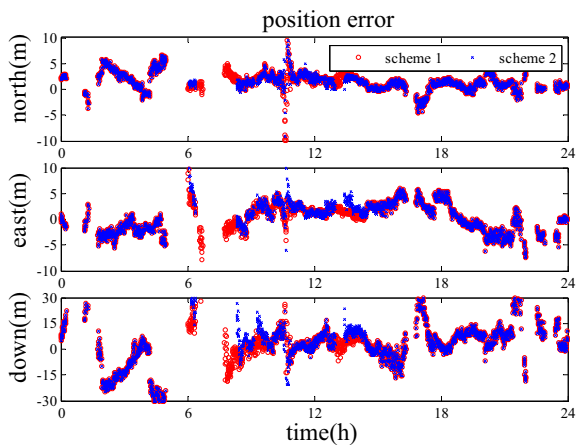

(e)

Fig. 6 SPP resolution of GPS/BDS system and GPS/BDS/QZSS system under different cut-off elevation angles at station CUT0 on February 5,2015: a $10^{\circ} ; \mathbf{b} 20^{\circ} ; \mathbf{c} 30^{\circ} ; \mathbf{d} 40^{\circ}$; e $50^{\circ}$

$$
\boldsymbol{\Phi}_{i f}=\frac{f_{1}^{2}}{f_{1}^{2}-f_{2}^{2}} \boldsymbol{\Phi}_{1}-\frac{f_{2}^{2}}{f_{1}^{2}-f_{2}^{2}} \boldsymbol{\Phi}_{2}=\rho+c \times\left(d t-d t_{s}\right)+T+\lambda_{i f} N_{i f}+M_{\boldsymbol{\Phi}}+\varepsilon_{\boldsymbol{\Phi}}
$$

where the subscript if is the ionosphere-free combination observation.

In order to test the improving availability and accuracy of GPS/BDS positioning using QZSS for single receiver, GPS, BDS and QZSS observation are process by single point positioning, static precise point positioning and kinematic precise point positioning, respectively. In single point positioning, only pseudorange is used ( $\mathrm{Li}$ et al. 2014). Both 
Table 2 The RMS and MAX values of SPP solution position error of GPS/BDS system and GPS/BDS/ QZSS system in north, east and down components

\begin{tabular}{|c|c|c|c|c|c|c|c|c|c|c|}
\hline \multirow[t]{2}{*}{ Cut-off elevation angle } & \multicolumn{2}{|c|}{$10^{\circ}(\mathrm{m})$} & \multicolumn{2}{|c|}{$20^{\circ}(\mathrm{m})$} & \multicolumn{2}{|c|}{$30^{\circ}(\mathrm{m})$} & \multicolumn{2}{|c|}{$40^{\circ}(\mathrm{m})$} & \multicolumn{2}{|c|}{$50^{\circ}(\mathrm{m})$} \\
\hline & RMS & MAX & RMS & MAX & RMS & MAX & RMS & MAX & RMS & MAX \\
\hline \multicolumn{11}{|l|}{ GPS/BDS/QZSS } \\
\hline North & 0.97 & 2.52 & 0.95 & 2.94 & 1.08 & 3.89 & 1.74 & 7.18 & 2.36 & 20.69 \\
\hline East & 0.71 & 2.31 & 0.84 & 2.42 & 1.14 & 2.92 & 1.95 & 6.96 & 2.61 & 10.49 \\
\hline Down & 3.02 & 8.82 & 3.10 & 9.68 & 3.93 & 12.43 & 7.85 & 22.38 & 12.91 & 41.36 \\
\hline \multicolumn{11}{|l|}{ GPS/BDS } \\
\hline North & 1.02 & 2.62 & 1.00 & 3.04 & 1.11 & 3.90 & 1.85 & 7.63 & 7.62 & 30.57 \\
\hline East & 0.74 & 2.31 & 0.87 & 2.42 & 1.16 & 2.99 & 2.03 & 6.96 & 3.42 & 19.64 \\
\hline Down & 3.19 & 9.06 & 3.19 & 9.87 & 3.98 & 13.33 & 8.67 & 25.92 & 21.50 & 56.33 \\
\hline
\end{tabular}

Table 3 The success rate of SPP solutions of GPS/BDS system and GPS/BDS/QZSS system

\begin{tabular}{llllll}
\hline Cut-off elevation angle & $10^{\circ}(\%)$ & $20^{\circ}(\%)$ & $30^{\circ}(\%)$ & $40^{\circ}(\%)$ & $50^{\circ}(\%)$ \\
\hline GPS/BDS/QZSS & 100 & 100 & 100 & 92.9 & 79.5 \\
GPS/BDS & 100 & 100 & 100 & 88.2 & 72.9 \\
\hline
\end{tabular}

pseudorange and carrier phase are used in static and kinematic precise point positioning. In kinematic precise point positioning, single point positioning resolution provides initial position to kinematic precise point positioning every epoch (Liu et al. 2015). The detailed resolution modes are shown in Fig. 2.

\section{Data analysis}

GNSS measurements recorded in 30-second intervals from IGS station CUT0 (Trimble NETR9 receiver) collected on February 5, 2015 are used in this study. The station CUT0 is located in Curtin, Australia. The final GNSS satellite orbits and clock products and the differential code biases produced by Center for Orbit Determination in Europe (CODE) are used. We also apply the absolute antenna phase center model and the phase windup corrections. The true coordinate benchmarks are from IGS weekly solution. The GPS/BDS/ QZSS observation was processed by SPP, static PPP and kinematic PPP mode, respectively. Figure $3 \mathrm{a}, \mathrm{b}$ and $\mathrm{c}$ show the sky plots (azimuth vs. elevation) of GPS, BDS and QZSS at station CUT0, respectively. The number of satellite for the various satellite systems under different cut-off elevation angles on that day are also calculated and shown in Fig. 4. Figure 5 shows the comparison of PDOP value under different cut-off elevation angles between GPS/BDS and GPS/BDS/QZSS. The PDOP value of three navigation system is less than that of GPS/BDS, especially under the cut-off elevation angles of $40^{\circ}$ and $50^{\circ}$.

\subsection{Single point positioning}

The legend of scheme 1 represents single point positioning method with GPS/BDS/QZSS and the legend of scheme 2 represents single point positioning method with GPS/BDS. 


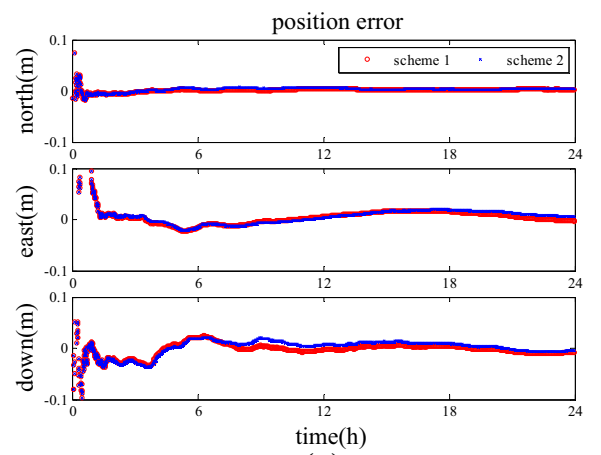

(a)

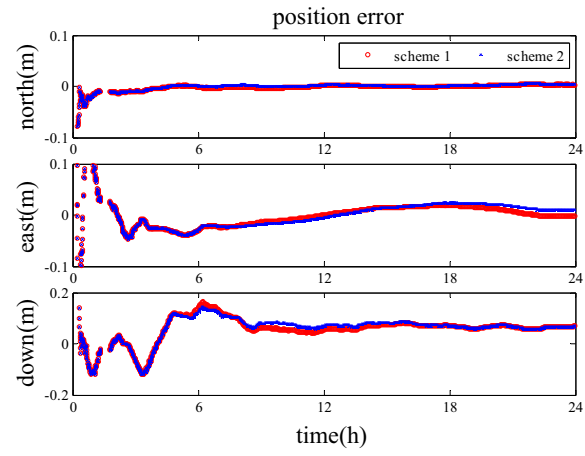

(c)
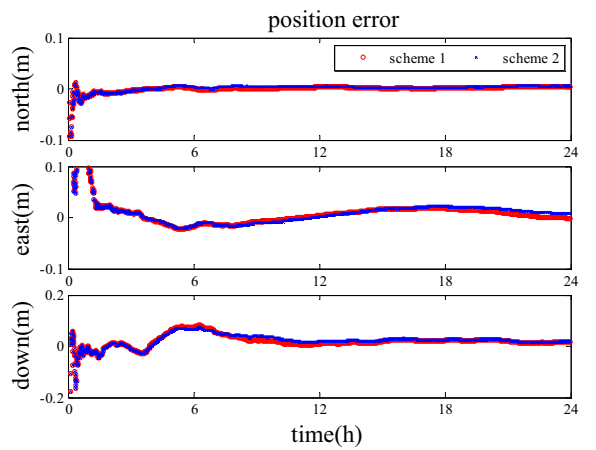

(b)

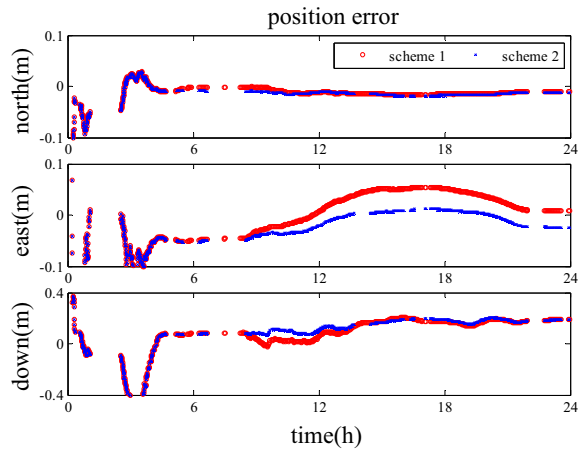

(d)

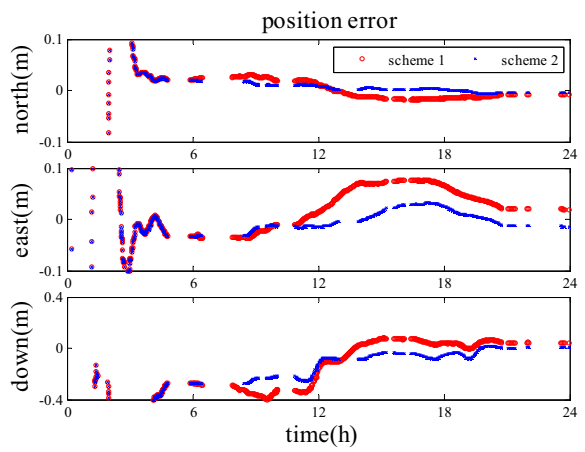

(e)

Fig. 7 Static PPP resolution of GPS/BDS system and GPS/BDS/QZSS system under different cut-off elevation angles at station CUT0 on February 5,2015 : a $10^{\circ}$; b $20^{\circ} ; \mathbf{c} 30^{\circ} ; \mathbf{d ~} 40^{\circ}$; e $50^{\circ}$

Position errors were computed with respect to the reference position to evaluate the performance. Figure 6 shows the time series of position errors in the north, east and down directions for scheme 1 and scheme 2. Table 2 illustrates root mean square errors (RMS) and maximum (MAX) value of position error. The success rate of SPP solutions of GPS/ BDS/QZSS system and GPS/BDS system is summarized in Table 3. Compared to scheme 2, scheme 1 reduces all the errors of position error in the north, east and down directions. When cut-off elevation angle is $50^{\circ}$, the position of scheme 2 can achieve an 
Table 4 The RMS and MAX values of static PPP solution position error of GPS/BDS system and GPS/ BDS/QZSS system in north, east and down components

\begin{tabular}{|c|c|c|c|c|c|c|c|c|c|c|}
\hline \multirow[t]{2}{*}{ Cut-off elevation angle } & \multicolumn{2}{|c|}{$10^{\circ}(\mathrm{m})$} & \multicolumn{2}{|c|}{$20^{\circ}(\mathrm{m})$} & \multicolumn{2}{|c|}{$30^{\circ}(\mathrm{m})$} & \multicolumn{2}{|c|}{$40^{\circ}(\mathrm{m})$} & \multicolumn{2}{|c|}{$50^{\circ}(\mathrm{m})$} \\
\hline & RMS & MAX & RMS & MAX & RMS & MAX & RMS & MAX & RMS & MAX \\
\hline \multicolumn{11}{|l|}{ GPS/BDS/QZSS } \\
\hline North & 0.003 & 0.006 & 0.003 & 0.004 & 0.003 & 0.007 & 0.012 & 0.016 & 0.036 & 0.070 \\
\hline East & 0.006 & 0.021 & 0.012 & 0.022 & 0.018 & 0.039 & 0.016 & 0.037 & 0.056 & 0.093 \\
\hline Down & 0.010 & 0.036 & 0.029 & 0.073 & 0.079 & 0.146 & 0.150 & 0.205 & 0.272 & 0.511 \\
\hline \multicolumn{11}{|l|}{ GPS/BDS } \\
\hline North & 0.004 & 0.007 & 0.004 & 0.005 & 0.004 & 0.007 & 0.014 & 0.018 & 0.065 & 0.096 \\
\hline East & 0.008 & 0.023 & 0.014 & 0.023 & 0.020 & 0.039 & 0.036 & 0.054 & 0.083 & 0.102 \\
\hline Down & 0.013 & 0.038 & 0.031 & 0.084 & 0.083 & 0.163 & 0.162 & 0.207 & 0.336 & 0.518 \\
\hline
\end{tabular}

Table 5 The success rate of static PPP solutions of GPS/BDS system and GPS/BDS/QZSS system

\begin{tabular}{llllll}
\hline Cut-off elevation angle & $10^{\circ}(\%)$ & $20^{\circ}(\%)$ & $30^{\circ}(\%)$ & $40^{\circ}(\%)$ & $50^{\circ}(\%)$ \\
\hline GPS/BDS/QZSS & 100 & 100 & 98.2 & 74.8 & 68.2 \\
GPS/BDS & 100 & 100 & 97.2 & 66.4 & 57.2 \\
\hline
\end{tabular}

accuracy of 2.36, 2.61 and $12.91 \mathrm{~m}$ in the north, east and down coordinate components, respectively. The success rate of SPP solutions of GPS/BDS system is improved from 88.2 to $92.9 \%$ using QZSS under $40^{\circ}$ cut-off elevation angle. At $50^{\circ}$ elevation cutoff, the QZSS improves the success rate of SPP solutions of GPS/BDS system from 72.9 to $79.5 \%$.

\subsection{Static precise point positioning}

The legend of scheme 1 represents static precise point positioning (PPP) method with GPS/ BDS/QZSS and the legend of scheme 2 represents static PPP method with GPS/BDS. Position errors were computed with respect to the reference position to evaluate the performance. Figure 7 shows the time series of position errors in the north, east and down directions for scheme 1 and scheme 2. Table 4 illustrates root mean square errors (RMS) and maximum (MAX) value of position error. The success rate of static PPP solutions of GPS/BDS/QZSS system and GPS/BDS system is summarized in Table 5. The same as SPP, compared to scheme 2, scheme 1 reduces all the errors of position error in the north, east and down directions. Compared with the scheme 2, the position accuracies in the north, east and down directions are improved by 44,33 and $19 \%$ for the scheme 1 under $50^{\circ}$ cut-off elevation angle, respectively. The success rate of static PPP solutions from GPS/BDS system is improved from 57.2 to $68.2 \%$ using QZSS under $50^{\circ}$ cut-off elevation angle.

\subsection{Kinematic precise point positioning}

The legend of scheme 1 represents kinematic precise point positioning (PPP) method with GPS/BDS/QZSS and the legend of scheme 2 represents kinematic PPP method with GPS/ BDS. Position errors were computed with respect to the reference position to evaluate the 

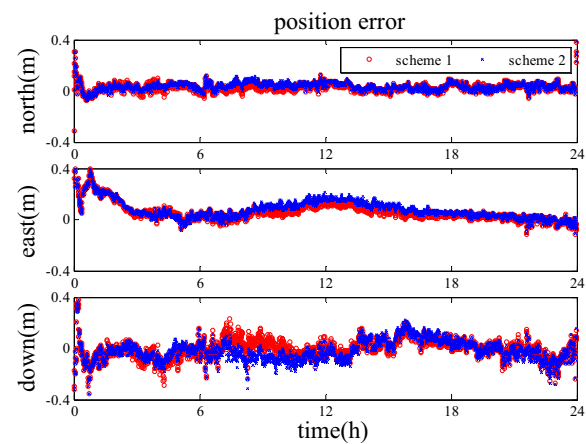

(a)
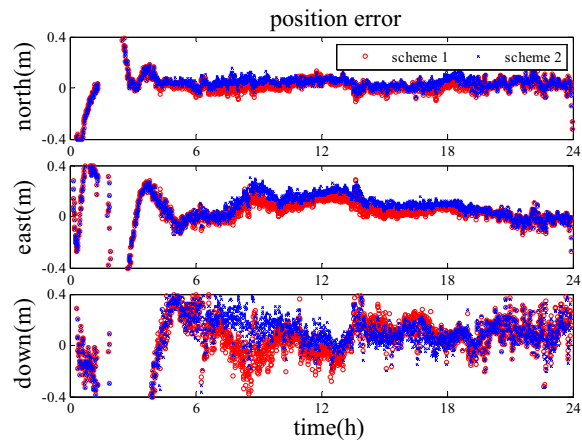

(c)
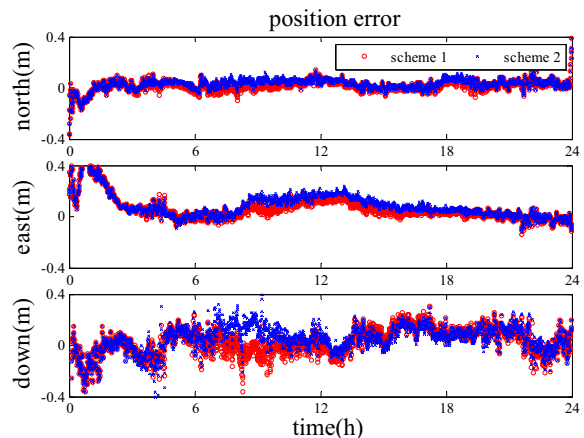

(b)

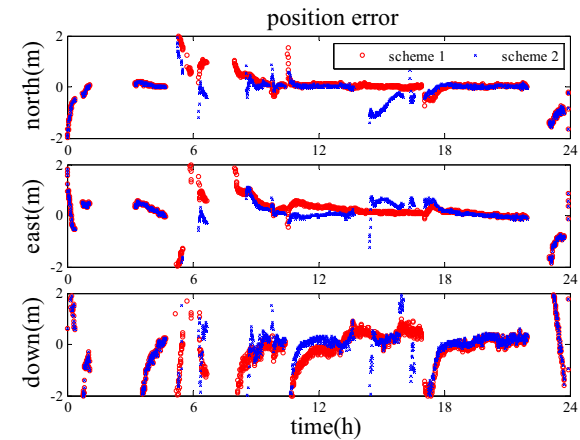

(d)

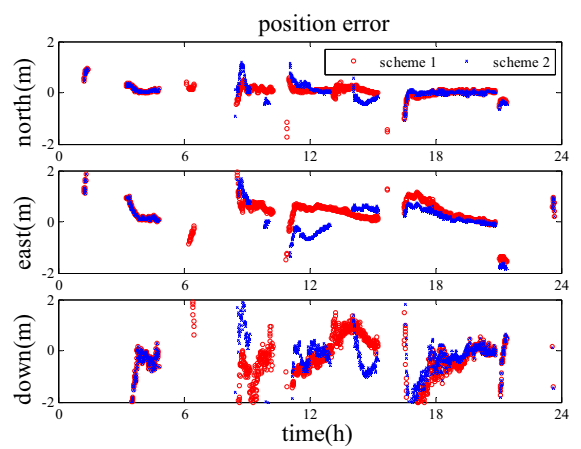

(e)

Fig. 8 Kinematic PPP resolution of GPS/BDS system and GPS/BDS/QZSS system under different cut-off elevation angles at station CUT0 on February 5,2015 : a $10^{\circ}$; b $20^{\circ}$; c $30^{\circ}$; d $40^{\circ}$; e $50^{\circ}$

performance. Figure 8 shows the time series of position errors in the north, east and down directions for scheme 1 and scheme 2. Table 6 illustrates root mean square errors (RMS) and maximum (MAX) value of position error. The success rate of static PPP solutions of GPS/BDS/QZSS system and GPS/BDS system is summarized in Table 7. Compared with the scheme 2, the position accuracies in the north, east and down directions are improved by 29,33 and $14 \%$ for the scheme 1 under $30^{\circ}$ cut-off elevation angle, respectively. The success rate of kinematic PPP solutions of GPS/BDS system is improved from 63.1 to 
Table 6 The RMS and MAX values of kinematic PPP solution position error of GPS/BDS system and GPS/BDS/QZSS system in north, east and down components

\begin{tabular}{|c|c|c|c|c|c|c|c|c|c|c|}
\hline \multirow[t]{2}{*}{ Cut-off elevation angle } & \multicolumn{2}{|c|}{$10^{\circ}(\mathrm{m})$} & \multicolumn{2}{|c|}{$20^{\circ}(\mathrm{m})$} & \multicolumn{2}{|c|}{$30^{\circ}(\mathrm{m})$} & \multicolumn{2}{|c|}{$40^{\circ}(\mathrm{m})$} & \multicolumn{2}{|c|}{$50^{\circ}(\mathrm{m})$} \\
\hline & RMS & MAX & RMS & MAX & RMS & MAX & RMS & MAX & RMS & MAX \\
\hline \multicolumn{11}{|l|}{ GPS/BDS/QZSS } \\
\hline North & 0.036 & 0.125 & 0.036 & 0.138 & 0.037 & 0.133 & 0.312 & 1.416 & 0.631 & 2.675 \\
\hline East & 0.073 & 0.232 & 0.072 & 0.185 & 0.089 & 0.286 & 0.353 & 0.672 & 0.589 & 1.457 \\
\hline Down & 0.066 & 0.286 & 0.114 & 0.363 & 0.160 & 0.554 & 0.860 & 3.864 & 1.369 & 4.946 \\
\hline \multicolumn{11}{|l|}{ GPS/BDS } \\
\hline North & 0.044 & 0.129 & 0.047 & 0.140 & 0.052 & 0.153 & 0.451 & 1.536 & 0.845 & 3.673 \\
\hline East & 0.095 & 0.232 & 0.102 & 0.238 & 0.133 & 0.302 & 0.416 & 1.228 & 0.966 & 2.962 \\
\hline Down & 0.073 & 0.311 & 0.102 & 0.402 & 0.185 & 0.734 & 0.895 & 4.904 & 1.762 & 6.326 \\
\hline
\end{tabular}

Table 7 The success rate of kinematic PPP solutions of GPS/BDS system and GPS/BDS/QZSS system

\begin{tabular}{llllll}
\hline Cut-off elevation angle & $10^{\circ}(\%)$ & $20^{\circ}(\%)$ & $30^{\circ}(\%)$ & $40^{\circ}(\%)$ & $50^{\circ}(\%)$ \\
\hline GPS/BDS/QZSS & 100 & 100 & 98.2 & 68.0 & 52.7 \\
GPS/BDS & 100 & 100 & 98.1 & 63.1 & 43.2 \\
\hline
\end{tabular}

$68.0 \%$ using QZSS under $40^{\circ}$ cut-off elevation angle. The success rate of kinematic PPP solutions of GPS/BDS system enhanced by QZSS is increased from $43.2 \%$ to $52.7 \%$ with $50^{\circ}$ elevation cutoff.

\section{Conclusion}

This paper presents GPS/BDS positioning using QZSS for single receiver. Through the analysis and comparison for the resolution error between GPS/BDS positioning and GPS/ BDS/QZSS positioning, the availability and accuracy of GPS/BDS positioning are improved with QZSS for single point positioning mode, static precise point positioning mode and kinematic precise point positioning mode. The QZSS is also able to increase the resolution success rate, especially in the period with high cut-off elevation angle. Compared with the GPS/BDS, the three-dimensional position accuracies of three resolution modes are improved by about $9 \%$ for the GPS/BDS/QZSS under $40^{\circ}$ cut-off elevation angle. The horizontal position from GPS/BDS/QZSS can achieve accuracy levels of 3.52, 0.067 and $0.863 \mathrm{~m}$ for three resolution modes under $50^{\circ}$ cut-off elevation angle, respectively. When compared to GPS/BDS, the GPS/BDS/QZSS improves the horizontal position accuracies by 58, 37 and $33 \%$, respectively. The success rate of SPP solutions from GPS/ BDS system is improved from 72.9 to $79.5 \%$, the success rate of static PPP solutions from GPS/BDS system is improved from 57.2 to $68.2 \%$ and the success rate of kinematic PPP solutions from GPS/BDS system is improved from 43.2 to $52.7 \%$ using QZSS under $50^{\circ}$ cut-off elevation angle. The success rate of GPS/BDS positioning is increased almost $10 \%$ with QZSS for single point positioning mode, static precise point positioning mode and kinematic precise point positioning mode under the cut-off elevation angles of $40^{\circ}$ and $50^{\circ}$. 
Acknowledgments The work is partially sponsored by the Fundamental Research Funds for the Central Universities (Grant Number: 2014ZDPY29) and partially sponsored by A Project Funded by the Priority Academic Program Development of Jiangsu Higher Education Institutions (Grant Number: SZBF2011-6B35).

\section{References}

Abdel-salam MA (2005) Precise point positioning using un-differenced code and carrier phase observations. The University of Calgary

Choy S, Harima K, Li Y, Wakabayashi Y, Tateshita H, Kogure S, Rizos C (2013) Real-time precise point positioning utilising the Japanese Quasi-Zenith Satellite System (QZSS) LEX corrections. In: International Global Navigation Satellite Systems Society IGNSS Symposium 2013, Outrigger Gold Coast, Qld Australia, July 16-18

Du S (2010) Integration of precise point positioning and low cost MEMS IMU. The University of Calgary

Harima K, Choy S, Wakabayashi Y, Kogure S, Rizos C (2014) Transmission of augmentation messages for precise point positioning utilizing Japanese QZSS LEX signal. In: Proceedings of the 27th International technical meeting of the ION satellite division, ION GNSS + 2014, Tampa, Florida, September 8-12

Hauschild A, Steigenberge P, Rodriguez-Solano C (2012) Signal, orbit and attitude analysis of Japan's first QZSS satellite Michibiki. GPS Solut 16:127-133

Hsu L, Gu Y, Kamijo S (2015) 3D building model-based pedestrian positioning method using GPS/ GLONASS/QZSS and its reliability calculation. GPS Solut. doi:10.1007/s10291-015-0451-7

Iwabuchi T, Rocken C, Wada A, Kanzaki M (2013) Benefit of multi GNSS processing with GPS,GLONASS, and QZSS for tropospheric monitoring. In: Proceedings of the 26th international technical meeting of the ION satellite division, ION GNSS + 2013, Nashville, Tennessee, September 16-20

Kitamura M, Ota T, Amano Y (2014) Improving availability and accuracy of multi-GNSS positioning using QZSS. In: Proceedings of the 27th international technical meeting of the ION satellite division, ION GNSS + 2014, Tampa, Florida, September 8-12

Landau H, Glocker M, Kipka A, Leandro R, Nitschke M, Stolz R, Zhang F (2012) Aspects of using the QZSS satellite in the Trimble CenterPoint ${ }^{\mathrm{TM}} \mathrm{RTX}^{\mathrm{TM}}$ service: QZSS orbit and clock accuracy, RTX positioning performance improvements. In: 25 th International technical meeting of the satellite division of the institute of navigation, Nashville TN, September 17-21

Li Y, Rizos C (2011) Evaluation of positioning accuracy of GNSS with QZSS augmentation. In: International global navigation satellite systems society IGNSS symposium 2011, Sydney, NSW, Australia, November $15-17$

Li Z, Wang J, Li B, Gao J, Tan X (2014) GPS/INS/Odometer integrated system using fuzzy neural network for land vehicle navigation applications. J Navig 67:967-983

Liu C, Lin P, Zhao X, Gao J (2015) Reducing GPS carrier phase errors in the measurement and position domains for short-distance static relative positioning. Acta Geod Geophys. doi:10.1007/s40328-0150111-y

Lukes Z et al. (2013) Network RTK computing in the cloud and the importance of using GLONASS and QZSS. In: Proceedings of the 26th International technical Meeting of the ION Satellite Division, ION GNSS + 2013, Nashville, Tennessee, September 16-20

Nadarajah N, Teunieesn PJG (2014) Instantaneous GPS/Galileo/QZSS/SBAS attitude determination: a single-frequency (L1/E1) robustness analysis under constrained environments. Navigation-US 61:65-75

Nadarajah N, Khodabandeh A, Teunissen PJG (2015) Assessing the IRNSS L5-signal in combination with GPS, Galileo, and QZSS L5/E5a-signals for positioning and navigation. GPS Solut. doi:10.1007/ s10291-015-0450-8

Odolinski R, Teunissen PJG, Odijk D (2014) Combined GPS + BDS + Galileo + QZSS for long baseline RTK positioning. In: Proceedings of the 27th International Technical Meeting of the ION Satellite Division, ION GNSS + 2014, Tampa, Florida, September 8-12

Odolinski R, Teunissen PJG, Odijk D (2015) Combined BDS Galileo, QZSS and GPS single-frequency RTK. GPS Solut 19:151-163

Su X, Zhan X, Niu M, Zhang Y (2014) Receiver autonomous integrity monitoring (RAIM) performances of combined GPS/BeiDou/QZSS in urban canyon. IEEJ T Electr Electr 9:275-281

Wu F, Kubo N, Yasuda A (2004) Performance analysis of GPS augmentation using Japanese quasi-zenith satellite system. Earth Planets Space 56:25-37 
Yamada H, Fujita S, Saitoh S, Sakai T, Ito K (2012) Real-time simulation of QZSS regional satellite navigation. In: 25th International Technical meeting of the satellite division of the institute of navigation, Nashville TN, September 17-21

Yoo BK, Sung S, Lee E, Lee S, Kim J, Lee H-J, Lee YJ (2009) Availability assessment of GPS augmentation system using geostationary gatellite and QZSS in seoul urban area. Japan Soc Aeronaut Space Sci 52:152-158 